CERN-ECP/95-03

27 January 1995

\title{
DAMAGE INDUCED BY PIONS IN SILICON DETECTORS
}

\author{
S.J. Bates, C. Furetta, M. Glaser, F. Lemeilleur, C. Soave \\ CERN, Geneva, Switzerland \\ E. León-Florián \\ University of Montreal, Montreal, Canada
}

RD2 Collaboration

\begin{abstract}
High-resistivity, ion-implanted silicon detectors have been irradiated with positive and negative pions up to fluences of $10^{14} \mathrm{~cm}^{-2}$ and $10^{13} \mathrm{~cm}^{-2}$, respectively. The energy dependence of the leakage-current damage constant around the $\Delta$ resonance is presented. Studies of the leakagecurrent damage constants (corrected for self-annealing and the long-term value) and the evolution of the depletion voltage with time show pions of momentum $350 \mathrm{MeV} / \mathrm{c}$ to be $20-25 \%$ more damaging than $1 \mathrm{MeV}$ neutrons. Charge-collection measurements are also presented that show no more degradation in efficiency than previously measured for $1 \mathrm{MeV}$ neutrons and $24 \mathrm{GeV} / \mathrm{c}$ protons.
\end{abstract}

Presented by S.J. Bates at the 4th International Conference on Advanced Technology and Particle Physics, Villa Olmo, Como, Italy, 3-7 October 1994. 
I he silicon detectors to be used at the tuture Large Hadron Coll1der (LHC) have to sustain a high tIux of particles. It is known that the damage induced in silicon by leptons is one to two orders of magnitude lower compared to hadrons [1]. Studies of the defects created in high-resistivity silicon material by neutrons and protons have already provided results. The diode reverse current increases as a function of the particle fluences, the effective doping concentration determining the full depletion voltage is modified, and the charge-collection efficiency is affected. More recently, hadron fluxes in the LHC detectors have been calculated [2], which point out the presence of a large flux of pions with a kinetic energy in the range $0.1-1 \mathrm{GeV}$ in comparison to other hadrons. Furthermore, the pion-induced damage in silicon could be enhanced around $200 \mathrm{MeV}$ due to the $\Delta$ resonance which leads to a larger $\pi$-nucleus total cross-section at this energy [3], and therefore necessitates a pion irradiation study to be performed.

The high-flux pion beam line of the Paul Scherrer Institute (PSI) at Villigen was used for the irradiation. This paper presents experimental results concerning the evolution of the electrical characteristics and chargecollection efficiency of single-diode silicon detectors irradiated with positive pions with a momentum of 350 $\mathrm{MeV} / c$ (close to the $\Delta$ resonance energy) up to a fluence of $10^{14} \mathrm{~cm}^{-2}$. Results obtained with negative pions as a function of the fluence are also presented. In addition, and in order to confirm the possible enhancement of the damage due to the $\Delta$ resonance, irradiations were performed with positive pions at various momenta from 150 to $450 \mathrm{MeV} / \mathrm{c}$.

\section{IRRADIATION DETAILS}

The pion irradiations were performed at the Paul Scherrer Institute, Villigen, $\mathrm{CH}$, using the high-intensity $\pi \mathrm{E} 1$ beam line set up specifically for the SIRAD collaboration. A double-magnet spectrometer allowed selection of positive and negative particles with momenta between 150 and $450 \mathrm{MeV} / c$. Carbon plates with masses ranging from 1 to $13 \mathrm{~g} \mathrm{~cm}^{-2}$, for use at different momenta, were inserted at the end of the beam line to stop protons and antiprotons. The contamination of neutrons with an energy greater than $500 \mathrm{keV}$, measured by indium activation foils, was less than $0.8 \%$ of the pion beam [4]. The lepton contamination could be neglected owing to the fact that the displacement damage in silicon is at least one order of magnitude less than for neutrons [1], and also the lepton contamination was at most $10 \%$.

An ionization chamber was used to monitor the pion-beam intensity and an XY chamber to measure the transverse beam profile along the beam axis at the irradiation positions. The pion fluence measurements were performed during the irradiation using aluminium activation foils with the same area as the silicon detectors and placed just in front of the detectors. The $\gamma(1369 \mathrm{keV})$ decay activity of ${ }^{24} \mathrm{Na}$, produced by the reaction ${ }^{27} \mathrm{Al}\left(\pi^{ \pm}, \mathrm{xN}\right){ }^{24} \mathrm{Na}$, was measured by a $\gamma$-spectrometer. From the activities of the aluminium samples, knowing the reaction cross-section at various momenta and the time of irradiation, the pion fluences were calculated [4]. The absolute fluence error is estimated to be of the order of $\pm 5 \%$.

For a cyclotron proton intensity of $\sim 750 \mu \mathrm{A}$, the $\pi^{+}$flux at the focus point was $\sim 7 \times 10^{8} \mathrm{~cm}^{-2} \mathrm{~s}^{-1}$ for momenta of 250, 300 and $350 \mathrm{MeV} / c$. At momenta of 150 and $400 \mathrm{MeV} / c$ the flux was $\sim 2 \times 10^{8} \mathrm{~cm}^{-2} \mathrm{~s}^{-1}$ and at $450 \mathrm{MeV} / c$ the flux was $\sim 1 \times 10^{8} \mathrm{~cm}^{-2} \mathrm{~s}^{-1}$. The $\pi^{-}$flux at $350 \mathrm{MeV} / c$ was a factor of 10 lower than the $\pi^{+}$ flux at the same momentum. The irradiations were performed at $25^{\circ} \mathrm{C}$, which was the temperature of the irradiation zone, and the silicon detectors used were $\sim 9 \mathrm{k} \Omega \mathrm{cm}$-type ion-implanted single diodes with a thickness of $300 \mu \mathrm{m}$ manufactured by Micron Semiconductor Ltd., UK.

The induced damage in the silicon bulk was studied first as a function of $\pi^{+}$momenta between 150 and 450 $\mathrm{MeV} / \mathrm{c}$ in order to look for the possible enhancement of the damage at the $\Delta$ resonance. For each momentum two $5 \times 5 \mathrm{~mm}^{2}$ active-area detectors were used and the fluence achieved was of the order of $10^{12} \mathrm{~cm}^{-2}$. Then two pion accumulation runs were performed, both at a pion momentum of $350 \mathrm{MeV} / c$, one with positive pions up to a fluence of $10^{14} \mathrm{~cm}^{-2}$ and the other with negative pions up to a fluence of $10^{13} \mathrm{~cm}^{-2}$, above the silicon conductivity type inversion point in both cases. For each of the accumulation runs, three detectors with an active area of $10 \times 10 \mathrm{~mm}^{2}$ were exposed in successive fluence steps and measured after each step. In addition, at each step of the irradiation during the $\pi^{+}$accumulation run, the collection efficiency of the charge deposited by relativistic electrons from a ${ }^{106} \mathrm{Ru}$ radioactive source was measured.

\section{RESULTS}




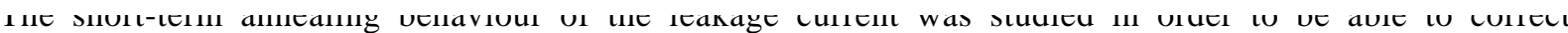
measurements made during irradiations of diodes lasting up to a few days. A single diode was irradiated in a positive pion beam of momentum $350 \mathrm{MeV} / \mathrm{c}$ to a fluence of $\phi=(8.59 \pm 0.26) \times 10^{11} \mathrm{~cm}^{-2}$. During the $26-\mathrm{minute}$ irradiation and for 48 hours following, the diode was reverse-biased at $80 \mathrm{~V}$ and the leakage current was continuously monitored. The depletion voltage, $26 \mathrm{~V}$ before irradiation, was found to be $20 \mathrm{~V}$ at the end and so the overbias applied varied only slightly in a region where the current-voltage characteristic normally shows a fairly flat behaviour. The temperature was monitored in the irradiation area and was found to vary $\pm 1^{\circ} \mathrm{C}$ around $25^{\circ} \mathrm{C}$. Since the leakage current $I_{l}$ is strongly dependent on temperature, the current was normalized to $25^{\circ} \mathrm{C}$ using the equation

$$
I_{l} \propto T^{3 / 2} e^{-E_{g} / 2 k T}
$$

where $E_{g}$ is the silicon band gap energy $\left(E_{g}=1.12 \mathrm{eV}\right.$ at room temperature), $T$ is the temperature in kelvin and $k$ is Boltzmann's constant. Figure 1 shows the leakage current during the 48 hours following the irradiation; the leakage current has been normalized to the first measurement and is hence the 'unannealed fraction'. The reduction is due to self-annealing of the radiation-induced defects which gave rise to the initial increase in leakage current. The annealing behaviour can be described, in this case, by the sum of four exponentials. It is obvious the annealing is not complete at the end of the 48 hours; however, the aim of this exposure was to parametrize the short-term annealing so that corrections could be made for other irradiations lasting about this amount of time. The solid line shown in Figure 1 is a fit according to:

$$
\frac{I_{t^{\prime}}}{I_{T_{l}}}=\sum_{i=1}^{4} A_{i} e^{-t^{\prime} / \tau_{i}}
$$

where $I_{t^{\prime}}$ is the leakage current at a time $t^{\prime}$ minutes after the end of the irradiation, $I_{l}$ is the leakage current at the end of the irradiation (of length $T_{l}$ minutes) and the fitted parameters $A_{i}$ and $\tau_{i}$ are given in Table 1 . The table also shows the values obtained by Aarnio et al. [5] after a pion irradiation at PSI in the same running period. They fitted more exponentials owing to a longer measurement time, but, the three smallest time constants for both sets of data are in good agreement.

In order to compensate for self-annealing, the procedure given in Ref. [6] is used, where the current which would be measured if no annealing during or after irradiation had taken place, $I_{0}$, can be calculated using:

$$
\frac{I_{t^{\prime}}}{I_{0}}=\sum_{i=1}^{n} A_{i} \frac{\tau_{i}}{T_{l}}\left[1-\exp \left(-\frac{T_{l}}{\tau_{i}}\right)\right] \exp \left(-\frac{t^{\prime}}{\tau_{i}}\right)
$$

Annealing corrections using both sets of parameters shown in Table 1 were performed, and to provide further comparison the neutron annealing parameters from Ref. [7] were also used. The results showed that the parameters obtained here and those presented in Ref. [7] gave very good agreement and the parameters obtained by Aarnio et al. showed a systematically larger correction factor of about $4 \%$. This difference is considered small enough that only the results obtained with the annealing corrections using our parameters are presented. 


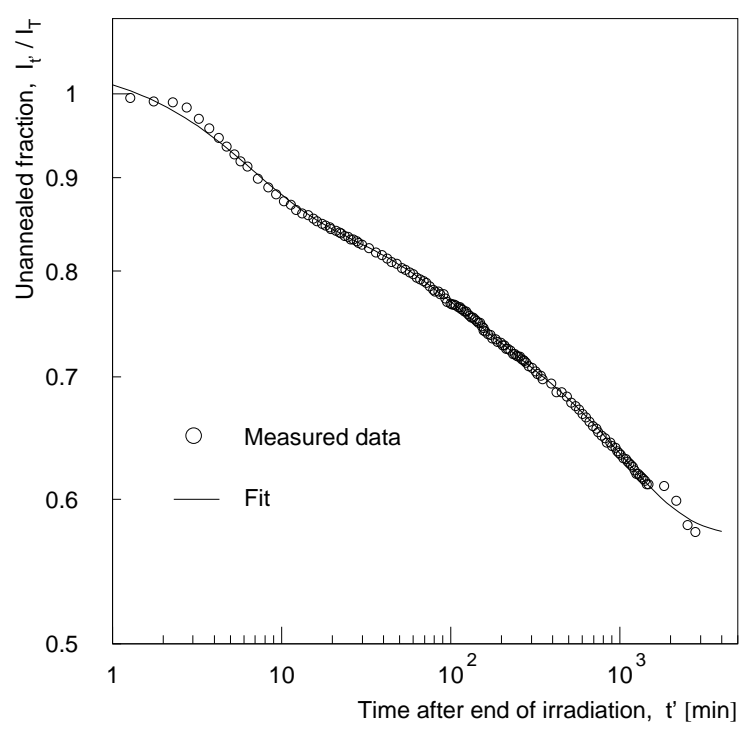

Figure 1. The short-term annealing of the leakage current, normalized to the first measurement, for 48 hours following a short (26-minute) irradiation. The solid line is a fit to the sum of four exponentials.

Table 1

The fitted parameters for the leakage current self-annealing compared to other pion data

\begin{tabular}{cccc}
\hline \multicolumn{2}{c}{ Data from this article } & \multicolumn{2}{c}{ Data from Ref. [5] } \\
\hline$A_{i}$ & $\tau_{i}[\mathrm{~min}]$ & $A_{i}$ & $\tau_{i}[\mathrm{~min}]$ \\
\hline $0.175 \pm 0.007$ & $5.55 \pm 0.30$ & 0.04 & 4.93 \\
$0.110 \pm 0.005$ & $80 \pm 4$ & 0.21 & 72 \\
$0.183 \pm 0.005$ & $922 \pm 31$ & 0.22 & 1043 \\
$0.574 \pm 0.030$ & $\infty$ & 0.19 & 21167 \\
& & 0.34 & $\infty$ \\
\hline
\end{tabular}

\subsubsection{Energy-dependence of damage}

An estimate of the displacement damage induced by pions, given in Ref. [3], predicts the same order of magnitude of damage as for $1 \mathrm{MeV}$ neutrons. However, at an energy of around $200 \mathrm{MeV}$, the resonance leads to a larger cross-section for interaction and so possibly to enhanced bulk damage. To look for evidence of this resonance, an energy scan was performed from $65 \mathrm{MeV}$ to $331 \mathrm{MeV}$ (corresponding to momenta of 150-450 $\mathrm{MeV} / c)$.

Short irradiations were performed with a positive pion beam to fluences of the order of $10^{12} \mathrm{~cm}^{-2}$. For each energy two detectors were irradiated and measured immediately after the irradiation. Owing to different fluxes at the various energies, the irradiations took between 30 minutes and 6 hours, therefore self-annealing corrections to leakage currents were used to compensate for different amounts of annealing. The leakage current was taken at full depletion and used to calculate the damage constant $\alpha$ according to the equation:

$$
\alpha=\frac{\Delta I}{\phi}
$$

where $\Delta I$ is the change in the volume leakage current. All currents were normalized to $20^{\circ} \mathrm{C}$ using Eq. (1) to compensate for slight differences in temperature during measurement. To take into account the self-annealing during and after irradiation, Eq. (3) was applied and Table 2 shows a summary of the results. Figure 2 shows the variation of damage constant with kinetic energy. Unfortunately, the error bars were larger than expected 


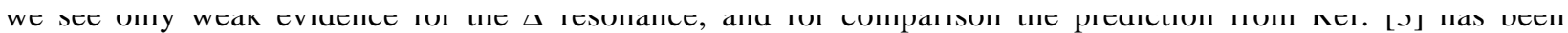
normalized to these data and plotted as the solid line in Figure 2. The variation expected over this range is certainly consistent with an enhancement of the damage at a kinetic energy close to $200 \mathrm{MeV}$.

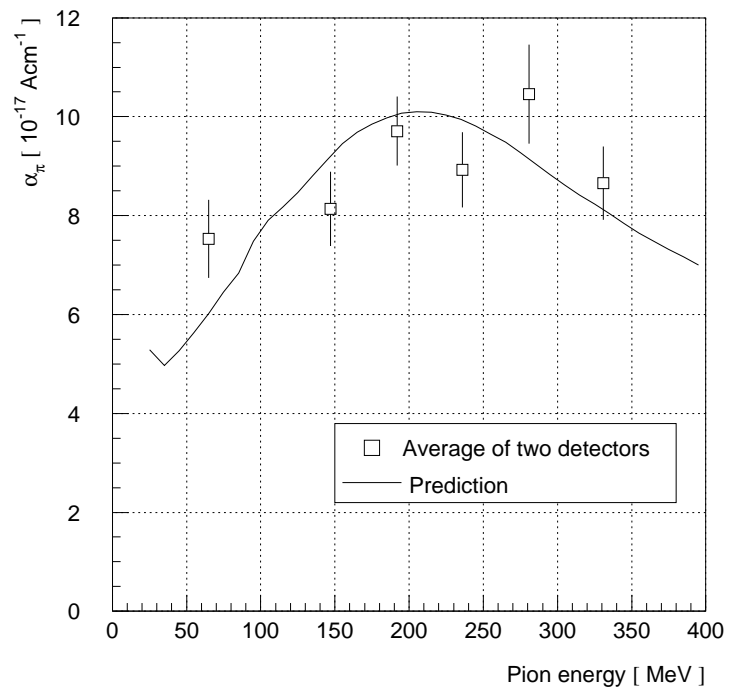

Figure 2. The variation of leakage-current damage constant with positive pion kinetic energy. The damage constants have been corrected for self-annealing and normalized to $20^{\circ} \mathrm{C}$.

Table 2

The results of the pion energy scan, the values of $\alpha$ have been corrected for self-annealing

\begin{tabular}{|c|c|c|c|c|c|c|}
\hline $\begin{array}{c}p \\
{[\mathrm{MeV} / c]}\end{array}$ & $\begin{array}{c}T \\
{[\mathrm{MeV}]}\end{array}$ & $\begin{array}{c}\phi \\
{\left[10^{12} \mathrm{~cm}^{-2}\right]}\end{array}$ & Detector & $\begin{array}{c}\alpha \\
10^{-17} \mathrm{Acm}^{-1}\end{array}$ & $\begin{array}{c}\text { Averages } \\
\alpha\end{array}$ & $1 \sigma$ \\
\hline 150 & 65 & $1.22 \pm 0.18$ & $\begin{array}{l}\text { MS1 } \\
\text { MS3 }\end{array}$ & $\begin{array}{l}8.0 \\
7.1\end{array}$ & 7.5 & 0.8 \\
\hline 250 & 147 & $1.9 \pm 0.24$ & $\begin{array}{l}\text { MS14 } \\
\text { MS15 }\end{array}$ & $\begin{array}{l}8.5 \\
7.8\end{array}$ & 8.1 & 0.7 \\
\hline 300 & 192 & $2.5 \pm 0.25$ & $\begin{array}{l}\text { MS11 } \\
\text { MS12 }\end{array}$ & $\begin{array}{l}9.8 \\
9.6\end{array}$ & 9.7 & 0.7 \\
\hline 350 & 236 & $2.48 \pm 0.29$ & $\begin{array}{l}\text { MS16 } \\
\text { MS17 }\end{array}$ & $\begin{array}{l}8.4 \\
9.5\end{array}$ & 8.9 & 0.8 \\
\hline 400 & 281 & $1.21 \pm 0.16$ & $\begin{array}{l}\text { MS8 } \\
\text { MS9 }\end{array}$ & $\begin{array}{l}10.0 \\
10.9\end{array}$ & 10.5 & 1.0 \\
\hline 450 & 331 & $1.2 \pm 0.14$ & $\begin{array}{l}\text { MS18 } \\
\text { MS20 }\end{array}$ & $\begin{array}{l}8.2 \\
9.1\end{array}$ & 8.7 & 0.7 \\
\hline
\end{tabular}

\subsubsection{Leakage-current damage constant}

To provide useful information for the LHC, it is desirable to study pion fluences up to at least $10^{14} \mathrm{~cm}^{-2}$, and to look for differences between positive and negative pions. As time was limited at PSI it was decided to study the positive pion accumulation at the pion momentum with highest flux, namely $350 \mathrm{MeV} / \mathrm{c}$, close in fact to the $\Delta$ resonance. For comparison, negative pions were studied at the same momentum albeit with a flux a factor of 10 lower. The fluxes for positive and negative pions were $5 \times 10^{8} \mathrm{~cm}^{-2} \mathrm{~s}^{-1}$ and $5 \times 10^{7} \mathrm{~cm}^{-2} \mathrm{~s}^{-1}$, respectively, lower than the maximum flux given above since our detectors were placed just in front of the focus point of the beam. In each study three similar diodes were irradiated in steps of increasing fluence with measurements immediately after each step. 


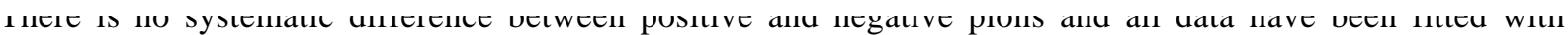
the form $\Delta I=\alpha \phi$ to give the damage constant. The damage constant was found to be $\alpha=(8.8 \pm 0.2) \times 10^{-17}$ $\mathrm{Acm}^{-1}$. This value is in excellent agreement with that found during the energy scan at the same momentum, see Table 2. In addition, it appears that the damage caused by these pions is very similar to that caused by $1 \mathrm{MeV}$ neutrons, where damage constants of the order of $8 \times 10^{-17} \mathrm{Acm}^{-1}$ are reported, albeit with a spread of values [8]. Aarnio et al. [5] report slightly higher pion damage constants of $\alpha=(10.4 \pm 0.7) \times 10^{-17} \mathrm{Acm}^{-1}, \alpha=$ $(12.2 \pm 0.5) \times 10^{-17} \mathrm{Acm}^{-1}$, and $\alpha=(10.7 \pm 0.7) \times 10^{-17} \mathrm{Acm}^{-1}$ for pion momenta of 200, $300 \mathrm{and} 400 \mathrm{MeV} / c$, respectively, and Dortmund reports a value of $10.5 \times 10^{-17} \mathrm{Acm}^{-1}$ [9]. So it appears that independent measurements vary a little; however, the damage-constant value corrected for self-annealing is very sensitive to the irradiation and measurement conditions and is of somewhat academic interest, therefore the emphasis is usually placed on the long-term, or stable damage constant, described next.

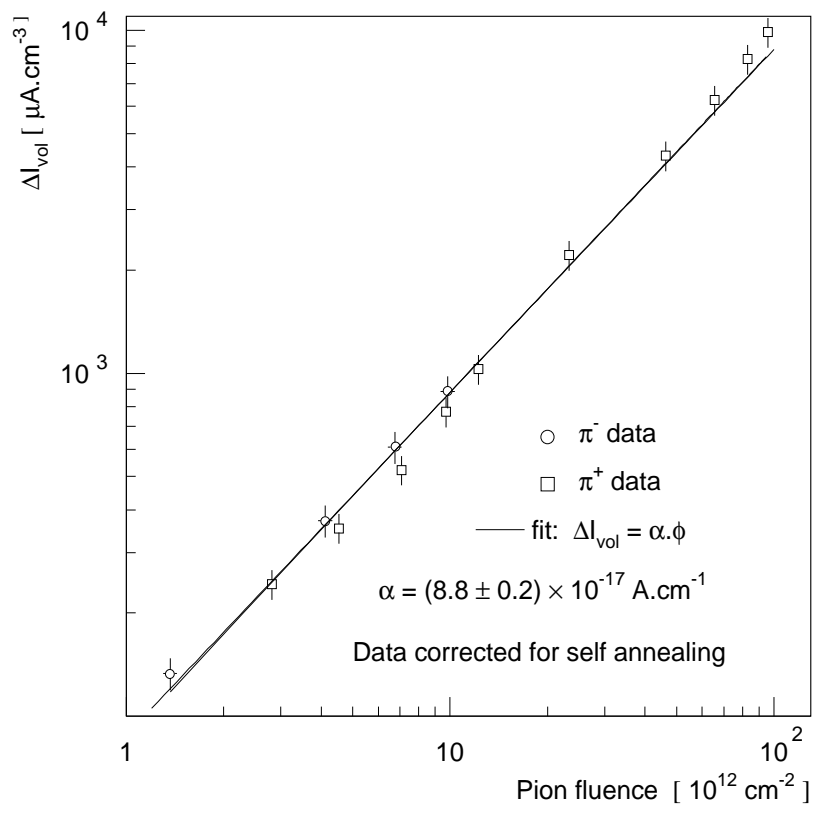

Figure 3. The change in volume leakage current versus $350 \mathrm{MeV} / c$ pion fluence. Leakage currents have been corrected for self-annealing and normalized to $20^{\circ} \mathrm{C}$. The solid line is a fit to all the points. 


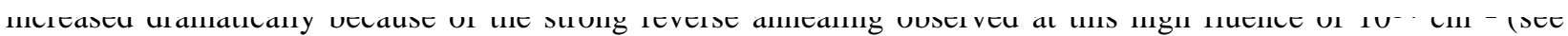
later). The depletion voltage actually exceeds the point at which the leakage current increases rapidly-an effect often termed 'breakdown' but which may be a combination of effects such as increased surface current or carrier injection. The positive pion data do not therefore represent the true damage constant value but instead indicate the danger of large depletion voltages.

Considering only the long term for negative pion data indicates a stable damage constant of $\alpha_{\text {stable }}=3.5 \times$ $10^{-17} \mathrm{Acm}^{-1}$. The annealing of the leakage current at room temperature is practically complete in the time shown and no significant further decrease is expected. The long-term value of $\alpha$ presented here is in good agreement with the values presented in Ref. [5] of $3.6 \times 10^{-17}$ to $4.1 \times 10^{-17} \mathrm{Acm}^{-1}$ which was measured 50 days after irradiations with momenta from 200 to $400 \mathrm{MeV} / c$. Dortmund report a value of $(3.56 \pm 0.04) \times 10^{-17}$ $\mathrm{Acm}^{-1}$ [9], and Hamburg an average value of $3.9 \times 10^{-17} \mathrm{Acm}^{-1}$ [10], both in good agreement with our data. The stable damage constant for pions of this limited momentum range is thus about $25 \%$ higher than the generally accepted value of $\sim 3 \times 10^{-17} \mathrm{Acm}^{-1}$ for $1 \mathrm{MeV}$ neutrons.

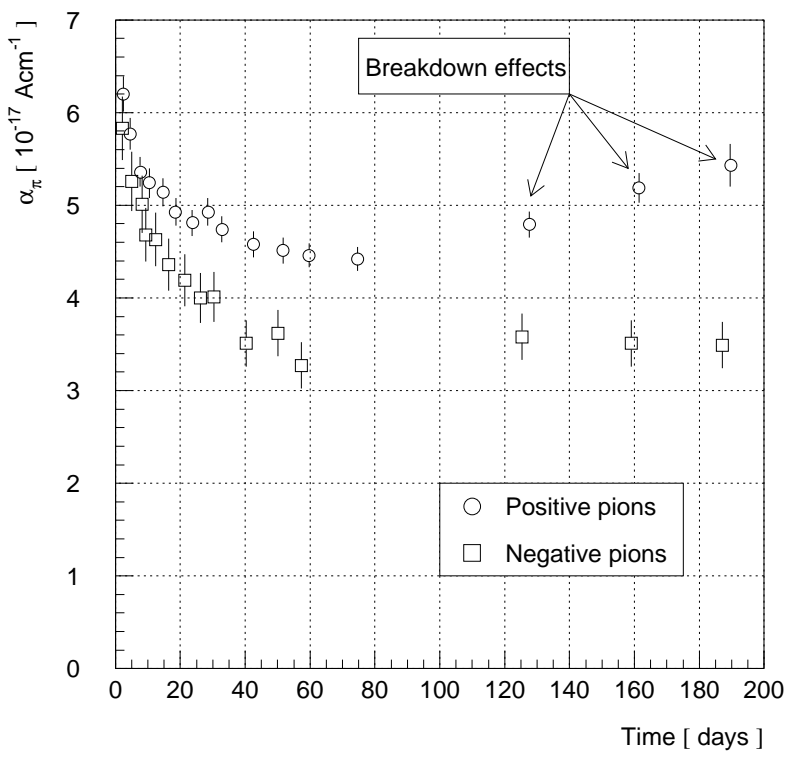

Figure 4. The leakage-current damage constant as a function of time after irradiation at room temperature.

\subsection{Depletion voltage}

\subsubsection{Effective impurity concentration, $N_{\text {eff }}$}

The absolute value of the effective impurity concentration is calculated from the depletion voltage using the equation:

$$
\left|N_{e f f}\right|=\left(V_{f d}+V_{b i}\right) \frac{2 \varepsilon_{r} \varepsilon_{0}}{q d_{0}^{2}},
$$

where $V_{f d}$ is the full depletion voltage determined from C-V curves, see Chapter 2 of Ref. [11], $V_{b i}$ is the junction built-in voltage $(\sim 0.6 \mathrm{~V}), \varepsilon_{r}$ is the relative permittivity of silicon, $\varepsilon_{0}$ the permittivity of free space, $q$ the electron charge and $d_{0}$ the thickness of the detector. Figure 5 shows the change in effective doping concentration with pion fluence. The squares show one detector irradiated with positive pions up to a fluence of $\phi_{\pi^{+}}=9.6 \times 10^{13} \mathrm{~cm}^{-2}$, while the circles show one detector irradiated with negative pions up to a fluence of $\phi_{\pi^{-}}=9.8 \times 10^{12} \mathrm{~cm}^{-2}$. Self-annealing corrections cannot be made in this case as the depletion voltage was not monitored during any irradiation. Only the positive pion data have been fitted with the functional form: 
where $N_{D, 0}$ is the initial concentration of donors, $N_{A, 0}$ is the initial concentration of acceptors, $c$ determines the rate of donor removal and $\beta$ the rate of acceptor creation. The initial concentration of doping, $N_{\text {eff }, 0}$ is given by:

$$
N_{e f f, 0}=N_{D, 0}-N_{A, 0},
$$

and since $N_{e f f, 0}$ is known, then only either $N_{D, 0}$ or $N_{A, 0}$ has to be a free parameter in the fit. The values of $N_{D, 0}$ and $N_{A, 0}$ are not generally given by the manufacturer, or easily determined.

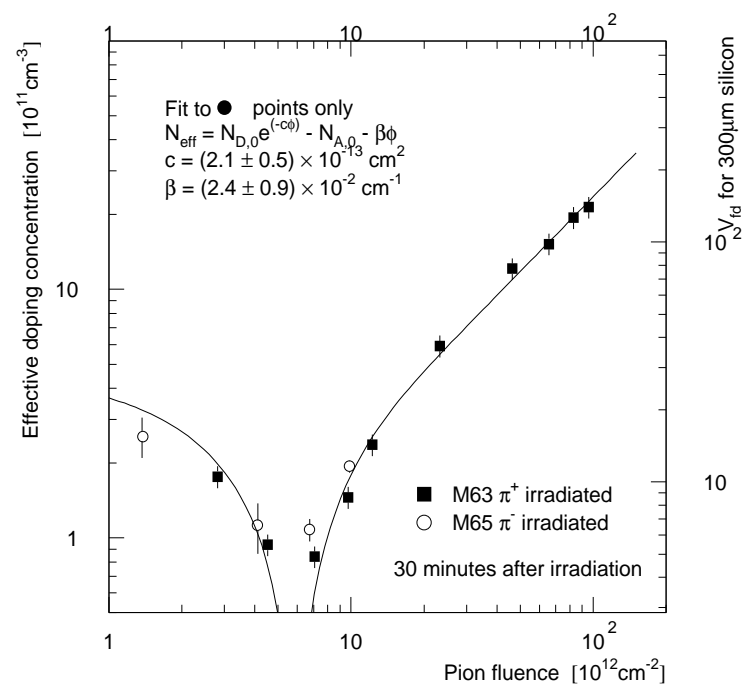

Figure 5. The absolute value of effective doping concentration calculated from depletion voltage as a function of $350 \mathrm{MeV} / \mathrm{c}$ pion fluence. The solid line is a fit to the positive pion points only.

The data follow the functional form of Eq. (6) well indicating that any annealing correction would be relatively small or affect all points systematically. The function goes through zero, indicating type inversion of the bulk, which is an effect common to neutron and proton irradiations of similar fluences. Also, there appears to be very little difference between the positive and negative pion data. The value of the constant $c$ is not very tightly bound owing to the small number of points before type inversion, but one can compare the value of $\beta=$ $(2.4 \pm 0.9) \times 10^{-2} \mathrm{~cm}^{-1}$ with that following proton and neutron irradiations performed at CERN. For example, in Ref. [12] we find for $1 \mathrm{MeV}$ neutron irradiations at a flux of $5.8 \times 10^{8} \mathrm{~cm}^{-2} \mathrm{~s}^{-1}$ (the same as for the positive pions) a somewhat lower value of $\beta=(1.4 \pm 0.1) \times$ $10^{-2} \mathrm{~cm}^{-1}$. For $24 \mathrm{GeV} / c$ protons with a flux 10 times higher we find a value of $\beta=(2.8 \pm 0.1) \times 10^{-2} \mathrm{~cm}^{-1}$. However, comparing values of $\beta$ from different irradiations with different conditions gives at best only an indication of the relative damage. In practice it is more useful to look at the long-term annealing behaviour which will be apparent during the LHC operation. 
Figure 0 shows the change in the depletion voltage at $2 U^{\sim} \mathcal{L}$ in the six months following the pion irradiation. Firstly the positive pion data shows very strong reverse annealing after an initial annealing of about two weeks. This has been observed following neutron and proton irradiations [12,13]. In the case of the negative pion data, reverse annealing is apparent but at a much slower rate due to the lower pion fluence received. Since the reverse annealing is not complete at this stage and can take several years to saturate at room temperature, it was decided to heat one positive-pion-irradiated detector to speed up the reverse annealing. Other work [14] predicts that heating at $110^{\circ} \mathrm{C}$ for one hour accelerates the annealing to $98 \%$ of the saturation value. For a detector of $300 \mu \mathrm{m}$ thickness, after a pion fluence of $10^{14} \mathrm{~cm}^{-2}$ and after heating, a depletion voltage of approximately $490 \mathrm{~V}$ was measured. This value can be compared with predicted values of about $420 \mathrm{~V}$ for 1 $\mathrm{MeV}$ neutrons and about $500 \mathrm{~V}$ for $24 \mathrm{GeV} / \mathrm{c}$ protons ${ }^{1}$. Therefore, in terms of the saturation value of depletion voltage, $350 \mathrm{MeV} / \mathrm{c}$ positive pions are about $20 \%$ more damaging than $1 \mathrm{MeV}$ neutrons.

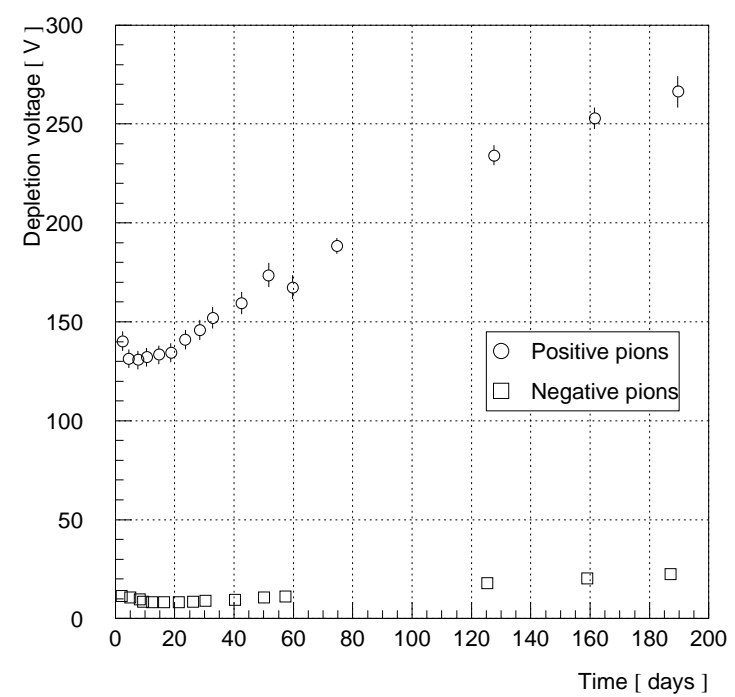

Figure 6. The depletion voltage versus time at room temperature after $350 \mathrm{MeV} / c$ positive and negative pion irradiations.

\subsection{Charge-collection efficiency}

Charge-collection measurements were performed using a ${ }^{106} \mathrm{Ru}$ source and scintillator trigger to select relativistic beta particles. The top trace in Figure 7a shows a typical current pulse from an unirradiated detector biased at $160 \mathrm{~V}$, where all the charge collected in less than $20 \mathrm{~ns}$. The bottom trace on this figure shows the integrated signal used to define the amount of charge collected, $100 \%$ in this case. Figure $7 \mathrm{~b}$ shows the same two traces for a detector irradiated with $350 \mathrm{MeV} / c$ positive pions to a fluence of $\phi=9.6 \times 10^{13} \mathrm{~cm}^{-2}$. The main observed effect is that the saturation value is less than $100 \%$. The charge-collection efficiency is thus defined as the charge collected in $20 \mathrm{~ns}$ after irradiation when normalized to the $100 \%$ value measured before irradiation. Measurements were made with at least $50 \mathrm{~V}$ of overbias. Figure 8 shows the charge-collection efficiency versus pion fluence. All measurements were made immediately after irradiation except for the highest fluence where measurements were made 74 days after irradiation. Owing to the reverse annealing of the depletion voltage, a bias of $245 \mathrm{~V}$ was necessary for this measurement. The charge-collection efficiency remains close to $100 \%$ until a fluence of about $3 \times 10^{13} \mathrm{~cm}^{-2}$ when it begins to fall off. However, even after a fluence close to $10^{14} \mathrm{~cm}^{-2}$ the charge collection efficiency is no less than $88 \%$ and considered sufficient for normal detector operation. This value is in exact agreement with that found after $24 \mathrm{GeV} / \mathrm{c}$ proton and $1 \mathrm{MeV}$ neutron irradiations to the same fluence $[12,13]$.

\footnotetext{
${ }^{1}$ Predictions were made using the second order reverse annealing model of the Hamburg group [14].
} 


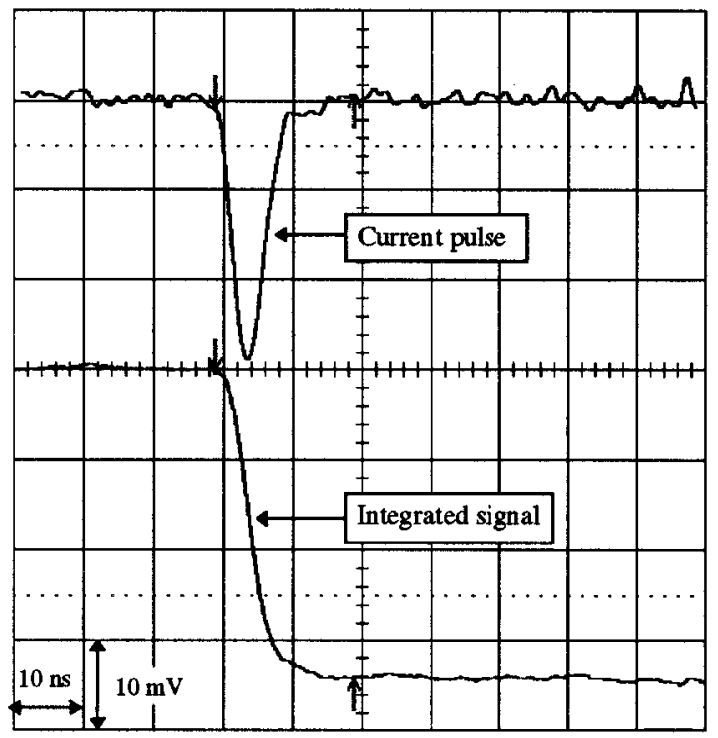

(a)

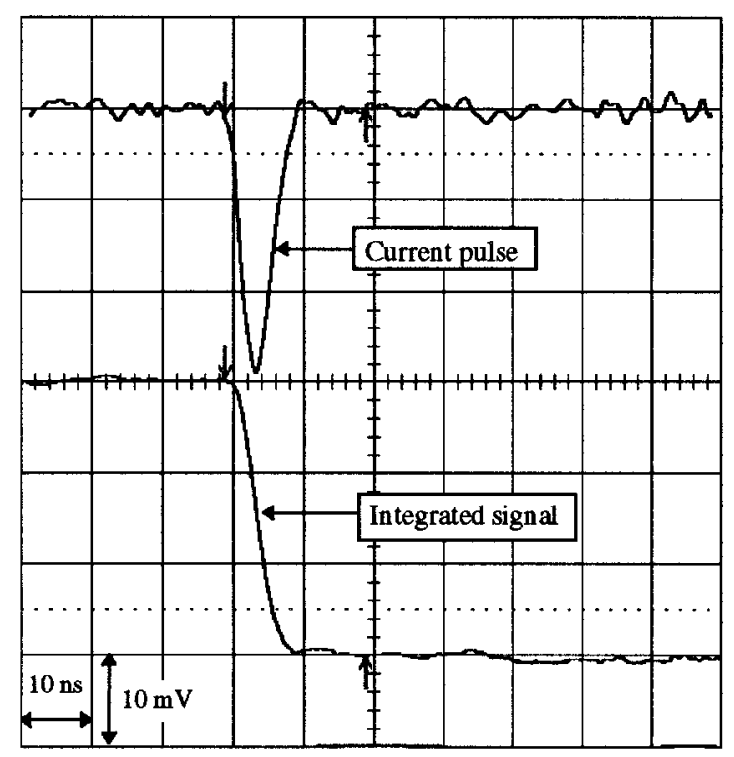

(b)

Figure 7. The typical current pulse response of a detector to relativistic electrons (top trace) and integrated signal (bottom trace) before irradiation (a) and after a $350 \mathrm{MeV} / c$ pion irradiation (b). 


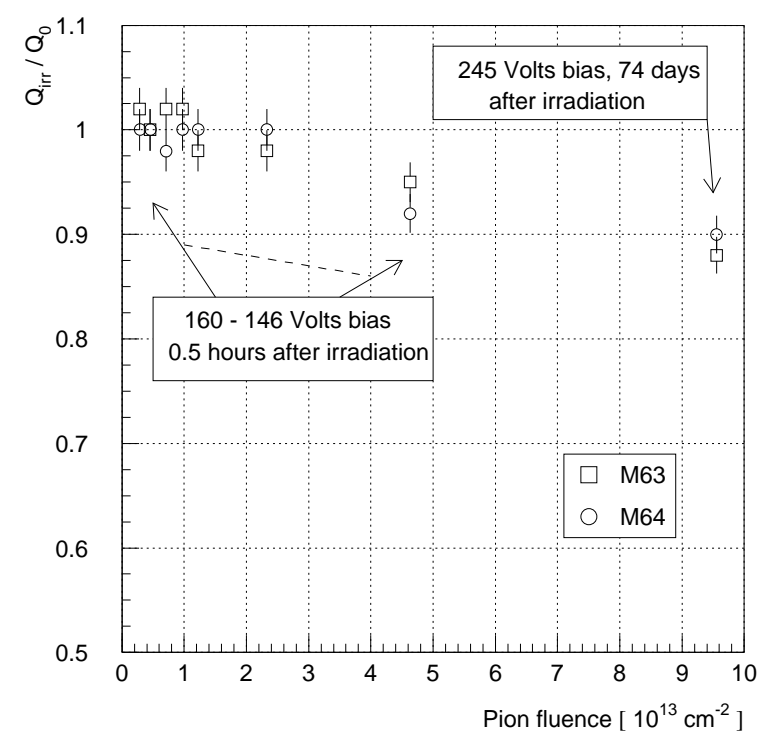

Figure 8. The charge-collection efficiency versus $350 \mathrm{MeV} / c$ positive pion fluence. The diodes were biased at least $50 \mathrm{~V}$ above the full depletion voltage and were measured immediately after irradiation except for the highest fluence where measurements were made 74 days after irradiation.

\section{CONCLUSIONS}

Pion irradiations were performed at PSI in order to measure the leakage-current damage constant and the change in depletion voltage, and to look for enhanced damage at the $\Delta$ resonance. A pion momentum scan was performed from 150 to $450 \mathrm{MeV} / c$ to relatively low fluence and then accumulation runs for positive and negative pions were performed at $350 \mathrm{MeV} / c$ to fluences of $\sim 10^{14} \mathrm{~cm}^{-2}$ and $\sim 10^{13} \mathrm{~cm}^{-2}$, respectively. Our results show only weak evidence for the $\Delta$ resonance, but are consistent with the relative variation predicted by Aarnio et al. [3].

The leakage-current damage constant measured at $350 \mathrm{MeV} / \mathrm{c}$ and corrected for self-annealing during the irradiation and before measurement was found to be $\alpha=(8.8 \pm 0.2) \times 10^{-17} \mathrm{Acm}^{-1}$. This value is slightly systematically lower than other values reported by Dortmund [9] and Aarnio et al. [5], however, the annealing corrected value of $\alpha$ is notoriously sensitive to exact irradiating conditions and measurement procedure due to rapid early annealing. There was no difference observed between positive and negative pions.

The stable damage constant measured 200 days after irradiation was found to be $\alpha=(3.5 \pm 0.2) \times$ $10^{-17} \mathrm{Acm}^{-1}$ which is in good agreement with measurements made by others $[5,9,10]$. The average value is approximately $25 \%$ higher than the commonly accepted value for $1 \mathrm{MeV}$ neutrons.

The depletion voltage showed the usual characteristic with particle fluence of reducing then increasing again after the conduction type inversion point. However, the reverse annealing at room temperature appears to be stronger for pions than for $1 \mathrm{MeV}$ neutrons. After 200 days of annealing at room temperature and after a fluence of $\sim 10^{14} \mathrm{~cm}^{-2}$, the depletion voltage was about $270 \mathrm{~V}$. After tempering one detector at $110^{\circ} \mathrm{C}$ for one hour the depletion voltage was about $490 \mathrm{~V}$, compared to a value of about $420 \mathrm{~V}$ predicted for $1 \mathrm{MeV}$ neutrons. Therefore, in terms of the saturation value of depletion voltage, $350 \mathrm{MeV} / c$ positive pions are about $20 \%$ more damaging than $1 \mathrm{MeV}$ neutrons.

The charge-collection efficiency was measured using a relativistic beta-particle source. The efficiency in an integration time of $20 \mathrm{~ns}$ after a pion fluence of $\sim 10^{14} \mathrm{~cm}^{-2}$ measured with $50 \mathrm{~V}$ of overbias was found to be $88 \%$. This value is exactly the same as measured after an irradiation with $24 \mathrm{GeV} / c$ protons and $1 \mathrm{MeV}$ neutrons to the same fluence [12]. 
I he authors wish to thank K. Horisberger, K. Gabathuler and K. Frosch of the Paul scherrer Institute tor their help in providing beam time, beam diagnostic instrumentation, and beam set-up. Our gratitude also goes to $\mathrm{C}$. Leroy of Montreal University for many important discussions.

\section{REFERENCES}

1. A. Van Ginneken, "Non-ionizing energy deposition in silicon for radiation damage studies", Fermi Lab. internal report FN-522, Oct. 1989.

2. P. Aarnio and M. Huhtinen, "Hadron fluxes in inner parts of LHC detectors", Nucl. Instrum. Methods A336 (1993) 98.

3. M. Huhtinen and P. Aarnio, "Pion induced displacement damage in silicon devices", Nucl. Instrum. Methods A335 (1993) 580.

4. C. Furetta, S.J. Bates, M. Glaser et al., CERN Detector R\&D Collaboration RD2, "Fluence and dosimetric measurements for a $\pi^{ \pm}$irradiation facility", Presented at teh 4th International Conference on Advanced Technology and Particle Physics, Villa Olmo, Como, Italy (1994). To be published in NIM.

5. P. Aarnio, M. Huhtinen, M. Pimiä et al., "Damage observed in silicon diodes after low energy pion irradiation", CMS TN/94-274 (1994).

6. G. Lindström, "Radiation damage in silicon detectors—self annealing corrections", SITP Internal Note, SITP-002 (1991).

7. R. Wunstorf, "Systematische Untersuchungen zur Strahlenresistenz von Silizium-Detektoren für die Verwendung in Hochenergiephysik-Experimenten", Ph.D. Thesis, DESY FH1K-92-01 (1992).

8. F. Anghinolfi, R. Bardos, S.J. Bates et al., CERN Detector R\&D Collaboration RD2, "Neutron irradiation of silicon diodes at temperatures of $+20^{\circ} \mathrm{C}$ and $-20^{\circ} \mathrm{C}$ ", Nucl. Instrum. Methods A326 (1993) 365-372.

9. Private Communication with A. Rolf, Dortmund University, Germany.

10. Private Communication with H. Feick, Hamburg University, Germany.

11. S.J. Bates, "The effects of proton and neutron irradiations on silicon detectors for the LHC", Ph.D. Thesis, Cambridge University, UK, RALT-006 (1994).

12. F. Lemeilleur, M. Glaser, E.H.M Heijne et al., CERN Detector R\&D Collaboration RD2, "Neutron, proton and gamma irradiations of silicon detectors", IEEE Trans. Nucl. Sci. 41 (1994) 425.

13. C. Leroy, M. Glaser, E.H.M Heijne et al., CERN Detector R\&D Collaboration RD2, "Study of electrical properties and charge collection of silicon detectors under neutron, proton and gamma irradiations", CERN/ECP 93-12, Proceedings of the 4th International Conference on Calorimetry in High-Energy Physics, La Biodola, Elba, Italy, 19-25 Sept. 1993, World Scientific, Singapore, 1994, p. 627.

14. H. Feick et al., CERN Detector R\&D Collaboration RD2, "Radiation studies and operational projections for silicon in the ATLAS inner detector", Proceedings of the 6th Pisa Meeting on Advanced Detectors, 1994, to be published in Nucl. Instrum. Methods Phys. Res. A. 